\title{
Diagnóstico de enfermagem Conhecimento Deficiente em usuárias de anticoncepcional oral combinado
}

\author{
Nursing diagnosis "Deficient Knowledge" in users of combined oral contraceptive
}

Ana Carolina Ribeiro Tamboril ${ }^{1}$, Jacqueline Alves da Silva Alcântara ${ }^{2}$, Aline Cruz Esmeraldo Áfio ${ }^{3}$, Carla Suellen Pires de Sousa ${ }^{4}$, Lidiane Nogueira Rebouças ${ }^{5}$, Escolástica Rejane Ferreira Moura ${ }^{6}$, Marcos Venícios de Oliveira Lopes ${ }^{7}$

${ }^{1}$ Enfermeira. Enfermeira Residente do Programa de Residência Integrada em Saúde da Escola de Saúde Pública do Ceará. Fortaleza, CE, Brasil. E-mail: tamboril_@hotmail.com.

${ }^{2}$ Enfermeira. Enfermeira da Secretaria de Saúde do Estado do Ceará. Fortaleza, CE, Brasil. E-mail: jac-alves2011@hotmail.com.

${ }^{3}$ Enfermeira, Mestre em Enfermagem. Professora da Faculdade Integrada da Grande Fortaleza. Fortaleza, CE, Brasil. E-mail: lineafio@hotmail.com.

${ }^{4}$ Enfermeira, Mestre em Enfermagem. Professora da Faculdade Vale do Jaguaribe. Aracati, CE, Brasil. E-mail: carlasusu6@hotmail.com.

${ }^{5}$ Enfermeira, Mestre em Enfermagem. Orientadora da Célula de Monitoramento e Avaliação da Secretaria Especial de Políticas sobre Drogas - SPD do

Governo do Estado do Ceará. Fortaleza, CE, Brasil. E-mail: lidianereboucas@hotmail.com.

${ }^{6}$ Enfermeira, Doutora em Enfermagem. Professor Associado da Universidade Federal do Ceará (UFC). Fortaleza, CE, Brasil. E-mail: escolpaz@yahoo.com.br.

${ }^{7}$ Enfermeiro, Doutor em Enfermagem. Professor Associado da UFC. Fortaleza, CE, Brasil. E-mail: marcos@ufc.br.

\section{RESUMO}

Objetivou-se analisar a acurácia das características definidoras do diagnóstico de enfermagem "Conhecimento Deficiente" em usuárias de AOC. Estudo transversal realizado com 97 mulheres em idade fértil em centro de saúde da família do nordeste brasileiro. A coleta de dados ocorreu de setembro a outubro de 2011, por meio de entrevista para identificação das características definidoras e dos fatores relacionados da Taxonomia II da NANDA-I 2012. O diagnóstico de enfermagem em questão apresentou prevalência de 58,8\%; a característica definidora "seguimento inadequado de instruções" (57,7\%) e o fator relacionado "interpretação errônea de informações” (58,8\%) foram os mais prevalentes. "Seguimento inadequado de instruções" apresentou maior sensibilidade, 96,61(88.46 - 99.07), elevado valor preditivo positivo (VPP=98,28) e elevado valor preditivo negativo (VPN=95,35). Todas as características definidoras apresentaram a mesma especificidade, 97,62(87.68 - 99.58). Conclui-se que as características definidoras estudadas apresentaram sensibilidade, especificidade, valores preditivos positivos e negativos altos para o diagnóstico de enfermagem analisado.

Descritores: Diagnóstico de Enfermagem; Conhecimento; Anticoncepcionais Orais Combinados.

\section{ABSTRACT}

Our objective was to analyze the accuracy of defining characteristics of the nursing diagnosis "Deficient Knowledge" in users of COC. A cross-sectional study conducted with 97 women in fertile age at a family health center in the Northeast Region of Brazil. The data collection was between September to October of 2011, through interviews to identify defining characteristics and factors related to Taxonomy II of NANDA-I 2012. The nursing diagnosis in question presented prevalence of $58.8 \%$; the defining characteristic "inadequate following of instructions" (57.7\%) and the related factor "wrong interpretation of information" were the most prevalent. "Inadequate following of instructions" presented higher sensitivity, 96.21 (88.46 - 99.07), high positive predictive value (PPV =98.28) and elevated negative predictive value (NPV=95.35). All defining characteristics presented the same specificity, 97.62 (87.68-99.58). It is concluded that studied defining characteristics presented high sensitivity, specificity, positive predictive values, and negative predictive values for the analyzed nursing diagnosis.

Descriptors: Nursing Diagnosis; Knowledge; Contraceptives, Oral, Combined. 


\section{INTRODUÇÃO}

$\mathrm{Na}$ contemporaneidade, há diversos métodos contraceptivos (MACs) disponíveis, os contraceptivos hormonais destacam-se entre os mais utilizados na população mundial. Nos últimos anos o uso de Anticoncepcionais Orais Combinados $(A O C)$ é crescente no Brasil ${ }^{(1)}$.

Pesquisa Nacional de Demografia e Saúde (PNDS) realizada em seis capitais brasileiras com 15.575 mulheres constatou que dentre as participantes com parceiro fixo $(82,4 \%), 80,5 \%$ estavam fazendo uso de algum método, sendo que $25,9 \%$ estavam laqueadas, $27,4 \%$ usavam anticoncepcional oral, $13 \%$ usavam preservativo masculino, 2,1\% praticavam o coito interrompido, 5,1\% tinham os parceiros vasectomizados e 7,0\% usam outros métodos como Dispositivo IntraUterino (DIU), diafragma, injeções, entre outros ${ }^{(2)}$.

Pílulas anticoncepcionais combinadas de baixa dose são constituídas de até $30 \mu \mathrm{g}$ de etinilestradiol em suas duas formas de apresentações: uma de 21 comprimidos, com pausa de sete dias entre uma cartela e a subsequente; e outra de 28 comprimidos, sendo de uso contínuo $^{(3)}$.

O AOC além de fornecer proteção segura, eficaz e reversível contra a gravidez, proporciona benéficos nãocontraceptivos, como controle do ciclo menstrual, alívio dos sintomas menstruais, melhora da saúde óssea, prevenção de ovário, endométrio e câncer colorretal e reduções na mortalidade materna e infantil. Salienta-se a presença de riscos e efeitos colaterais associados a esse método ${ }^{(4)}$. Nesse sentido a decisão contraceptiva deve ser baseada nos riscos e benefícios.

O uso correto e consistente do método contraceptivo é o principal determinante de sua eficácia. A adoção de comportamentos sexuais seguro por mulheres é um desafio para profissionais de saúde, pois a abordagem individualizada durante a consulta de Planejamento Familiar (PF) ainda não é contemplada por todos que estão envolvidos nesse processo ${ }^{(5)}$.
Estima-se taxa de falha de oito gravidezes para 100 usuárias por ano. Contudo, se não houver falhas no uso, menos de uma gravidez poderá ocorrer para 100 mulheres por ano. A taxa de falha do método está diretamente relacionada com o uso incorreto do mesmo, bem como a presença dos efeitos colaterais, fator importante para o abandono ${ }^{(3)}$.

Sabe-se que a educação em saúde é uma das principais ferramentas de cuidado de enfermeiros. Assim constata-se sua importância nas ações de promoção da saúde, especificamente no período preconcepção, promovendo o planejamento da gravidez ${ }^{(6)}$.

A promoção adequada do $A O C$ deve seguir recomendações da Organização Mundial da Saúde (OMS), para que ocorra decisão voluntária e esclarecida sobre métodos anticoncepcionais existentes. Desse modo mulheres que procuram o serviço de PF devem ser esclarecidas quanto a eficácia do método, seu modo de uso, como funciona, efeitos colaterais, riscos e benefícios para a saúde, sinais e sintomas indicativos de retorno ao serviço, retorno à fertilidade após a interrupção do uso e prevenção de Doenças Sexualmente Transmissíveis $(D S T s)^{(7)}$. Todas essas informações devem ser oferecidas às usuárias de $A O C$ nas consultas de Enfermagem.

A consulta de Enfermagem pressupõe aplicação da Sistematização da Assistência de Enfermagem (SAE) e permite o enfermeiro realizar levantamento de dados, estabelecer o Diagnóstico de Enfermagem (DE), prescrever e implementar intervenções e avaliar resultados $^{(8)}$. É definido pela NANDA Internacional, como um "julgamento clínico das respostas do indivíduo, família ou da comunidade aos processos vitais ou aos problemas de saúde atuais ou potenciais. Fornece a base para a seleção das intervenções de enfermagem”(9).

A NANDA Internacional define o DE Conhecimento Deficiente, como "a ausência ou deficiência de informação cognitiva relacionada a um tópico específico". Tem como características definidoras: comportamentos exagerados, comportamentos impróprios, desempenho inadequado em um teste, seguimento inadequado de 
instruções e verbalização do problema. Seus fatores relacionados são: falta de capacidade de recordar, falta de exposição, falta de familiaridade com os recursos de informação, falta de interesse em aprender, interpretação errônea de informações e limitação cognitiva $^{(9)}$.

Em face ao exposto, a pesquisa teve como objetivo analisar a acurácia das características definidoras do DE “Conhecimento Deficiente" em usuárias de AOC.

\section{MÉTODOS}

Estudo transversal, realizado em Centro de Saúde da Família (CSF) em um município do nordeste brasileiro. 0 referido CSF possui sete Equipes de Saúde da Família (ESF) para atender a uma população média de 28.000 pessoas.

A população do estudo correspondeu às mulheres em idade fértil (10 a 49 anos) adscritas ao CSF, sendo a amostra ( $n=97)$ calculada com base na fórmula para populações infinitas:

$$
n=\frac{Z_{\alpha}^{2} \cdot P \cdot(1-P)}{e^{2}}
$$

Onde, n: é o tamanho da amostra; Za: nível de significância em desvio-padrão (95\%); P: prevalência do fenômeno (50\%); E: erro amostral (10\%). Adotou-se o valor máximo de $P$ pela falta de informações sobre a proporção exata de mulheres em idade fértil que buscam o serviço.

Os critérios de inclusão foram: estar em idade fértil e ser acompanhada no referido CSF. Foram excluídas as mulheres que recebiam acompanhamento em outros CSFs ou em serviço privado de saúde.

A coleta de dados ocorreu em setembro e outubro de 2011, por meio de entrevista estruturada com finalidade de identificar fatores relacionados, sinais e sintomas que compõem as características definidoras do diagnóstico em estudo, de acordo com a Taxonomia II da NANDA-I ${ }^{(9)}$. As participantes foram abordadas nos diversos setores de atendimento do CSF, sendo que o instrumento da coleta de dados contemplou: (1) dados sociodemográficos; (2) informações relacionadas ao uso habitual do AOC; (3) teste acerca do conhecimento de mulheres quanto ao uso correto do método (Quadro 1).

As características definidoras e fatores relacionados foram conceituados pelos autores com base em critérios, clínicos e operacionais, estabelecidos em literatura específica ${ }^{(3)}$ e experiência dos mesmos com a temática em questão. Desse modo determinaram-se as definições pela concordância absoluta entre os autores.

A presença do DE "Conhecimento Deficiente" foi determinado de acordo com a presença de uma ou mais das características definidoras apresentadas no Quadro $1^{(10)}$.

Os dados foram organizados no software EXCEL for WINDOWS e analisados no SPSS versão 19.0. Para avaliar a associação entre as características definidoras e a presença do DE foram utilizados o Teste do Qui-quadrado de Pearson, para frequências esperadas maiores que cinco; e o teste da probabilidade exata de Fisher, para frequências menores que cinco. Quando as tabelas apresentaram mais do que duas categorias e frequências esperadas menores que cinco aplicou-se o teste de FisherFreeman-Halton. A magnitude da associação entre variáveis categóricas foi mensurada pela razão de prevalência. Para verificação de normalidade dos dados utilizou-se o teste de Shapiro-Wilk ou o teste de Kolmogorov-Smirnov. Para verificação de homogeneidade de variâncias foi aplicado o teste de Levene. Com base nos resultados destes testes aplicou-se o teste T ou de Mann-Whitney, para verificação de diferença de média / mediana entre os grupos.

Por fim, a análise da acurácia foi realizada com base nas medidas de sensibilidade, especificidade e probabilidades pós-teste (positiva e negativa) das características definidoras e dos fatores relacionados levantados no estudo. A sensibilidade representa a probabilidade de identificar corretamente a presença do indicador clínico em pacientes com o DE. A especificidade é a probabilidade de identificar corretamente a ausência do indicador clínico em pacientes sem o DE. O valor 
preditivo de uma característica clínica, quando positivo (VPP), representa a probabilidade de se encontrar o DE em pacientes que apresentem tal característica definidora. Quando negativo (VPN), representa a probabilidade da ausência do DE em pacientes sem tal característica definidora $^{(11)}$.
O projeto de pesquisa foi submetido e aprovado pelo Comitê de Ética em Pesquisa da Universidade Federal do Ceará, conforme protocolo no 160/11. Todas as participantes assinaram o Termo de Consentimento Livre e Esclarecido. O estudo seguiu as normas brasileiras para pesquisas com seres humanos.

Quadro 1: Definição das características definidoras e dos fatores relacionados ao DE - Conhecimento Deficiente do AOC.

\begin{tabular}{|c|c|}
\hline $\begin{array}{l}\text { Características } \\
\text { definidoras }\end{array}$ & Definições \\
\hline $\begin{array}{l}\text { Comportamentos } \\
\text { exagerados }\end{array}$ & Toma o AOC em excesso. Por exemplo: Toma mais de uma pílula por dia. \\
\hline $\begin{array}{l}\text { Comportamentos } \\
\text { impróprios }\end{array}$ & $\begin{array}{l}\text { Utiliza o AOC de forma incorreta, embora não exagerada. Representa uma conduta imprópria que, } \\
\text { apesar de não ser excessivamente intensa, pode levar a ocorrência de gravidez. Por exemplo: Não } \\
\text { toma a pílula todos os dias; Toma a pílula somente quando tem relação sexual; Após } \\
\text { esquecimento da tomada descarta o restante da cartela. }\end{array}$ \\
\hline $\begin{array}{l}\text { Desempenho inadequado } \\
\text { em um teste }\end{array}$ & $\begin{array}{l}\text { Desempenho inferior ao esperado ou desejado em uma avaliação de conhecimento. Considerou- } \\
\text { se inadequado quando a mulher responde incorretamente as quatro perguntas acerca do uso } \\
\text { correto de AOC. }\end{array}$ \\
\hline $\begin{array}{l}\text { Seguimento inadequado } \\
\text { de instruções }\end{array}$ & $\begin{array}{l}\text { Não cumprimento ou realização de atividades ou normas julgadas necessárias. Inclui qualquer } \\
\text { atitude da mulher que seja incongruente com as recomendações oferecidas pelo profissional. Por } \\
\text { exemplo: Não inicia a tomada do } \mathrm{AOC} \text { nos primeiros cinco dias do ciclo menstrual. }\end{array}$ \\
\hline Verbalização do problema & $\begin{array}{l}\text { Reconhece que possui o problema através de relato. Caracterizada pelo relato verbal de } \\
\text { dificuldade em usar corretamente o AOC (esquecimento constante, presença de efeitos colaterais, } \\
\text { não adaptação ao método). }\end{array}$ \\
\hline \multicolumn{2}{|l|}{ Fatores relacionados } \\
\hline $\begin{array}{l}\text { Falta de capacidade de } \\
\text { recordar }\end{array}$ & $\begin{array}{l}\text { Incapacidade de buscar na memória informações antes armazenadas relacionadas às orientações } \\
\text { recebidas. Dificuldade para lembrar das instruções de uso do AOC e/ou apresenta episódios de } \\
\text { esquecimento frequentes. }\end{array}$ \\
\hline Falta de exposição & Relata nunca ter utilizado o $\mathrm{AOC}$ antes. \\
\hline $\begin{array}{l}\text { Falta de familiaridade com } \\
\text { os recursos de informação }\end{array}$ & $\begin{array}{l}\text { Interpretação inadequada das orientações contidas na cartela, bula e/ou ofertada pelo } \\
\text { profissional. }\end{array}$ \\
\hline $\begin{array}{l}\text { Falta de interesse em } \\
\text { aprender }\end{array}$ & $\begin{array}{l}\text { Ausência de perguntas sobre a conduta a ser adotada e direcionamento da atenção a fatos ou } \\
\text { circunstâncias não relacionadas ao uso adequado do AOC. }\end{array}$ \\
\hline $\begin{array}{l}\text { Interpretação errônea de } \\
\text { informações }\end{array}$ & Divergência entre o que foi orientado pelo profissional e o que é executado pela cliente. \\
\hline Limitação cognitiva & $\begin{array}{l}\text { Dificuldade de leitura e/ou raciocínio demonstrados pela dificuldade de compreender as } \\
\text { instruções oferecidas. }\end{array}$ \\
\hline
\end{tabular}

\section{RESULTADOS}

No que concerne à escolaridade, predominou o ensino médio completo, sendo este referido por 61 mulheres (62,9\%). A ocupação "do lar" foi referida por 30 mulheres $(30,9 \%)$ e $27(27,8 \%)$ citaram "estudante". O status marital "casada” foi citado por 51 mulheres (52,6\%) (Tabela 1).

Ao avaliar a normalidade das variáveis socioeconômicas, com a utilização do teste KolmogorovSmirnov, obteve-se heterogeneidade relacionada a idade, renda familiar e tempo de uso do AOC, com isso adotouse a mediana.

As entrevistadas apresentaram mediana de idade de 24 anos $(I Q=7)$, renda familiar de dois salários $(I Q=1)$ e número de pessoas na família de quatro pessoas $(I Q=1)$. O tempo de uso do anticoncepcional oral obteve mediana de 48 meses $(I Q=72)$. 
Tabela 1: Distribuição do número de participantes segundo dados demográficos socioeconômicos e tempo de uso de AOC. Fortaleza, CE, Brasil, 2011.

\begin{tabular}{|c|c|c|c|c|c|}
\hline Variáveis (n=97) & & & & No. & $\%$ \\
\hline \multicolumn{6}{|l|}{ Escolaridade } \\
\hline Fundamental & & & & 10 & 10,3 \\
\hline Médio & & & & 84 & 86,5 \\
\hline Superior & & & & 3 & 3,2 \\
\hline \multicolumn{6}{|l|}{ Ocupação } \\
\hline Trabalho fora do lar & & & & 31 & 31,9 \\
\hline Do lar & & & & 30 & 30,9 \\
\hline Estudante & & & & 27 & 27,8 \\
\hline Outros & & & & 9 & 9,1 \\
\hline \multicolumn{6}{|l|}{ Condição de união } \\
\hline Casada ou em união consensual & & & & 51 & 52,6 \\
\hline Solteira & & & & 45 & 46,4 \\
\hline \multirow[t]{2}{*}{ Divorciada } & & & & 1 & 1 \\
\hline & Média & DP & Mediana & IQ & Valor de $p$ \\
\hline Idade (anos completos) & 24,08 & 5,082 & 24 & 7 & 0,021 \\
\hline Renda familiar (salário mínimo*) & 1,97 & 770 & 2 & 1 & $<0,001$ \\
\hline Tempo de uso de AOC (em meses) & 61,22 & 46,707 & 48 & 72 & $<0,001$ \\
\hline \multicolumn{6}{|l|}{ * DP: desvio padrão } \\
\hline \multicolumn{6}{|l|}{ * IQ: inter-quartis } \\
\hline \multicolumn{6}{|l|}{ *Valor de p: teste de Kolmogorov-Smirnov } \\
\hline * salário mínimo vigente na pesquisa $-\mathrm{R} \$ 545,00$ & & & & & \\
\hline
\end{tabular}

A presença de falha do método foi citada por 18 mulheres $(18,6 \%)$, sendo esta atribuída ao esquecimento na tomada diária. Referente à indicação do método 79 $(81,4 \%)$ citaram receber indicação de um profissional da saúde, destas 56 (70,9\%) citaram o médico e 23 (29,1\%) citaram o enfermeiro. Porém, $13(13,4 \%)$ admitiram que iniciaram o método por conta própria e cinco $(5,2 \%)$ referiram a indicação por amigos.
De acordo com a Tabela 2, verifica-se que a característica definidora "seguimento inadequado de instruções" foi a mais prevalente $56(57,7 \%)$, e o fator relacionado interpretação errônea de informações 57 (58,8\%). Em relação ao diagnóstico de enfermagem conhecimento deficiente, $57(58,8 \%)$ das usuárias o apresentaram.

Tabela 2: Prevalência das características definidoras e fatores relacionados do diagnóstico de enfermagem conhecimento deficiente. Fortaleza, CE, Brasil, 2011.

\begin{tabular}{|c|c|c|}
\hline Variáveis & No. & $\%$ \\
\hline \multicolumn{3}{|l|}{ Características Definidoras } \\
\hline Seguimento inadequado de instruções & 56 & 57,7 \\
\hline Verbalização do problema & 4 & 4,1 \\
\hline Comportamentos impróprios & 2 & 2,1 \\
\hline Desempenho inadequado em um teste & 1 & 1 \\
\hline \multicolumn{3}{|l|}{ Fatores Relacionados } \\
\hline Interpretação errônea de informações & 57 & 58,8 \\
\hline Falta de capacidade de recordar & 7 & 7,2 \\
\hline Falta de exposição & 4 & 4,1 \\
\hline Falta de interesse em aprender & 2 & 2,1 \\
\hline Diagnóstico de Enfermagem Conhecimento Deficiente & 57 & 58,8 \\
\hline
\end{tabular}

$\mathrm{Na}$ análise bivariada entre as características definidoras ou o diagnóstico de enfermagem e as variáveis clínicas e demográficas, não foi identificada associação estatisticamente significante.
A característica definidora "seguimento inadequado de instruções" apresentou associação significativa com o DE Conhecimento Deficiente $(p<0,001)$. Quanto aos fatores relacionados “interpretação errônea de 
informações" apresentou significância estatística $(p<0,001)$.

$\mathrm{Na}$ Tabela 3 observa-se que a característica definidora "Seguimento inadequado de instruções" foi a de maior sensibilidade, assim, esta apresenta maior probabilidade de identificar corretamente a presença do
DE em estudo nas usuárias de AOC. Tal característica também apresentou alto valor preditivo positivo $(\mathrm{VPP}=98,28)$ o que indica uma maior probabilidade da confirmação do DE conhecimento deficiente. Todas as características definidoras obtiveram a mesma especificidade.

Tabela 3: Medidas de acurácia das características definidoras do diagnóstico conhecimento deficiente. Fortaleza, CE, Brasil, 2011.

\begin{tabular}{|c|c|c|c|c|c|c|c|c|c|}
\hline $\begin{array}{ll}\text { Características } & \text { Variáveis } \\
\text { definidoras }\end{array}$ & $\mathbf{S}$ & $\mathbf{E}$ & VPP & VPN & RV+ IC (95\%) & RV- IC (95\%) & ORD IC (95\%) & ROC & p \\
\hline Comportamentos impróprios & 5,08 & 97,6 & 75 & 42,2 & $\begin{array}{c}2,14 \\
(0,30-15,05)\end{array}$ & $\begin{array}{c}0,97 \\
(0,90-1,05)\end{array}$ & $\begin{array}{c}2,01 \\
(0,22-59,5)\end{array}$ & 0,513 & 0,51 \\
\hline $\begin{array}{c}\text { Desempenho inadequado em um } \\
\text { teste }\end{array}$ & 3,3 & 97,6 & 66,6 & 41,8 & $\begin{array}{c}1,42 \\
(0,20-10,04)\end{array}$ & $\begin{array}{c}0,99 \\
(0,93-1,06)\end{array}$ & $\begin{array}{c}1,35 \\
(0,11-43,41)\end{array}$ & 0,505 & 0,4 \\
\hline $\begin{array}{l}\text { Seguimento inadequado de } \\
\text { instruções }\end{array}$ & 96,6 & 97,6 & 98,2 & 95,3 & $\begin{array}{c}40,58 \\
(0,30-15,05)\end{array}$ & $\begin{array}{c}0,03 \\
(0,01-0,14)\end{array}$ & $\begin{array}{c}778,06 \\
(107,77-16384)\end{array}$ & 0,971 & $<0,001$ \\
\hline Verbalização de problema & 8,47 & 97,6 & 83,3 & 43,1 & $\begin{array}{c}3,56 \\
(0,51-25,07)\end{array}$ & $\begin{array}{c}0,94 \\
(0,86-1,03)\end{array}$ & $\begin{array}{c}3,4 \\
(0,50-92,38)\end{array}$ & 0,53 & 0,14 \\
\hline
\end{tabular}

* Sensibilidade (S), Especificidade (E), Valor Preditivo Positivo (VPP), Valor Preditivo Negativo (VPN), Razão de Verossimilhança Positiva (RV+), Razão de Verossimilhança Negativa (RV-) , Odds Ratio diagnóstica (ORD) e Área sob a curva ROC (ROC)

* IC intervalo de confiança

* p teste Qui-quadrado de Pearson

As características seguimento inadequado de instruções e verbalização de problema apresentaram alto valor preditivo positivo e negativo, indicando uma elevada probabilidade de ocorrência do DE conhecimento deficiente na presença dessas características e ausência do referido diagnóstico quando usuárias de $A O C$ não apresentarem tais características. Os maiores valores de Área sob a curva ROC foram obtidos nas características seguimento inadequado de instruções $(0,971)$ e verbalização de problema $(0,530)$ (Tabela 3).

A análise odds ratio (ORD) diagnóstica verifica a probabilidade da ocorrência do diagnóstico de acordo com a presença da característica definidora, desse modo o seguimento inadequado de instruções foi o sinal clínico com maior chance de ocorrer. (Tabela 3).

\section{DISCUSSÃO}

O total de pessoas entre 18 e 20 anos com ensino médio mais que triplicou nas duas últimas décadas, com o indicador passando de $13 \%$ para $41 \%{ }^{(12)}$. Em discordância desse estudo onde mais da metade das participantes concluíram o ensino médio, condição que pode influenciar o conhecimento sobre o AOC.

Essa correlação foi constatada, por exemplo, em estudo realizado em Fortaleza-CE com 264 usuárias de AOC, em que os autores identificaram associação entre o uso correto do $\mathrm{AOC}$ e as variáveis escolaridade e renda familiar, sendo que para ambas houve correlação positiva, sugerindo que quanto maior a escolaridade $(r=0,164 ; p=0,008)$ e/ou a renda familiar $(r=0,135$; $p=0,029)$ maior o conhecimento das participantes sobre o uso correto de $\mathrm{AOC}^{(13)}$.

Em relação à ocupação, a porcentagem de mulheres com trabalho fora do lar foi praticamente igual a de mulheres exercendo apenas atividades do lar. Ratifica-se que a inclusão de mulheres no mercado de trabalho despertou interesse pela ascensão social, com consequente redução da fecundidade, enfatizando a necessidade da assistência ao planejamento familiar ${ }^{(14)}$.

Concernente à condição de união houve prevalência de mulheres casadas ou em união consensual. Em concordância com pesquisa realizada em centros de 
saúde de Fortaleza-CE que $84,1 \%$ das participantes referiram união estável ${ }^{(13)}$.

Houve predomínio de mulheres jovens no estudo, fato que consoante estudo pode inferir no conhecimento acerca de métodos anticoncepcionais em geral, haja vista que espera-se que pessoas de idade mais avançada apresentem maior senso de responsabilidade, o que pode conduzir a maior busca por informações e serviços de saúde e desejo de limitar a prole ${ }^{(15)}$.

A renda familiar média identificada no estudo caracteriza as participantes como classe social "pobre, mas não extremamente pobre" ${ }^{\prime(16)}$. Esperava-se tal resultado na ESF pesquisada, uma vez que a prioridade de implantação das equipes são os territórios de indicadores socioeconômicos menos favoráveis. Esta condição levou autores afirmarem que fatores socioeconômicos desfavoráveis devem ser enfatizados no processo de aconselhamento em planejamento familiar, no sentido de alertar as mulheres, homens e/ou casais quanto a decisão de ter ou não ter filhos e quando tê-los, em face da infraestrutura financeira da família ${ }^{(17)}$.

O tempo de uso do $A O C$ pode relacionar-se ao conhecimento que as usuárias possuem com relação ao método. Pesquisa sugere que mulheres que usaram $A O C$ por mais tempo conheciam mais sobre seus efeitos colaterais $(r=0,189 ; p=0,002)^{(13)}$. Estudo realizado com 1.427 mulheres entre 18 e 39 anos reforça a relação entre a presença de efeitos colaterais e a descontinuidade do método contraceptivo, em que $57,3 \%$ das participantes, após seis meses do início do uso de AOC, haviam descontinuado o uso devido aos efeitos colaterais ${ }^{(18)}$. Assim, percebe-se que o baixo conhecimento de usuárias de um método anticoncepcional poderá elevar o índice de abandono do método.

Estudo realizado na cidade de El Paso, Texas (EUA) com 108 mulheres identificou que $100 \%$ das que utilizam o AOC relatam ser inconveniente a tomada diária de pílulas anticoncepcionais, fato relacionado ao uso incorreto e inconsistente corroborando com falhas no uso do método ${ }^{(19)}$.
$\mathrm{Na}$ consulta de Enfermagem ao planejamento familiar deve-se investigar dificuldades da usuária de AOC em lembrar a tomada diária, auxiliando-a a encontrar meios fáceis e acessíveis que facilitem esse processo de memorização, como: manter o medicamento em local estratégico a ser visualizado no horário da tomada; relacionar a tomada a alguma atividade diária; envolver o parceiro no sentido de lembrar à usuária; utilizar um despertador disponível, como do telefone móvel, dentre outros.

Indicação de métodos contraceptivos por profissionais da saúde corrobora o uso correto. Estudo realizado com 273 adolescentes reforça essa assertiva, pois ao identificar como principal fonte de informação os pais e a mídia, $75 \%$ tinham conhecimento muito escasso sobre os MACs ${ }^{(20)}$.

De encontro ao resultado da presente pesquisa o déficit de conhecimento sobre o AOC foi identificado em estudo realizado em Teresina-PI, envolvendo 278 adolescentes no qual foi aplicado um questionário e escala para avaliar o conhecimento objetivo acerca do uso correto do AOC. Identificou-se que $98,2 \%$ dos participantes tinham baixo conhecimento objetivo, ou seja, acertaram três itens ou menos da escala ${ }^{(21)}$.

Em contrapartida, estudo realizado em Fortaleza-CE identificou resultado mais positivo ao verificar $50 \%$ das participantes com conhecimento substancial ou extenso sobre o uso correto e os efeitos colaterais do AOC. No geral, o conhecimento foi limitado apenas sobre as complicações associadas ao uso do $\mathrm{AOC}^{(13)}$.

Houve predominância da característica definidora "seguimento inadequado de instruções", enfatizando a dificuldade que as usuárias apresentaram em compreender totalmente as instruções fornecidas pelos profissionais de saúde. Tais limitações se concentraram na forma correta de iniciar o método e no que fazer frente ao esquecimento da tomada diária do AOC. Esses achados corroboram com estudo realizado com 357 mulheres na Arábia Saudita, apontando alto índice de conhecimento inadequado sobre o uso do AOC em 63,9\% das 
participantes $^{(22)}$. Dados preocupantes, pois o uso do método de forma inconsistente e incorreta pode resultar em uma gravidez não planejada.

Quanto aos fatores relacionados o de maior prevalência nesse estudo foi "interpretação errônea de informações". Fato que reflete a fragilidade da assimilação das orientações fornecidas pelos profissionais da saúde às mulheres. É de suma importância a avaliação da qualidade das orientações fornecidas, pois outros fatores, como a compreensão das informações, memória e nível de educação das mulheres, podem influenciar no conhecimento $^{(22)}$.

Resultados positivos foram alcançados ao longo prazo na Alemanha ao aplicar materiais escritos em 124 mulheres com designo de aumentar conhecimento sobre AOC, ressalta-se que esta estratégia possibilitou mudanças na atitude e prática das usuárias ${ }^{(23)}$.

A especificidade das características definidoras contribui para prática da enfermagem ao auxiliar a identificação do DE durante as consultas de planejamento familiar. Salienta-se que o enfermeiro deve estar atento para identificar usuárias que não se sentem a vontade para expor dúvidas ou dificuldades em relação ao método de anticoncepção. Essa peculiaridade da atuação da enfermagem contribuirá na identificação precoce das características definidoras, em particular a verbalização do problema ${ }^{(24)}$.

A análise da acurácia do diagnóstico de enfermagem conhecimento deficiente reforçou evidências de diversos estudos, que identificaram baixa qualidade das informações sobre o uso correto do $A O C$ e a dificuldade

\section{REFERÊNCIAS}

1. United Nations. Departamento of Economic and Social Affaiirs, Population Division: 2011. World contraceptive use 2010.(POP/DBCPrec 2010).

2. Ministério da Saúde. Pesquisa Nacional de Demografia e Saúde da Criança e da Mulher - PNDS 2006: dimensões do processo reprodutivo e da saúde da criança. Brasília (Brasil): Ministério da Saúde; 2009. 300p. de compreensão destas pelas usuárias ${ }^{(13-22-25)}$. Percebe-se que a presença do diagnóstico em estudo nas usuárias de AOC pode influenciar na eficácia do método e torna-as mais susceptíveis a uma gravidez não planejada.

\section{CONCLUSÃO}

A análise da acurácia das características definidoras do diagnóstico de Enfermagem "Conhecimento Deficiente" em usuárias de AOC identificou que apesar do elevado percentual de mulheres com aspectos positivos que favorecem apreensão do conhecimento sobre o método, como orientações providas por profissionais (médico ou enfermeiro) a respeito do AOC e considerável nível de escolaridade (ensino médio completo), ainda permanecem dificuldades por parte do grupo pesquisado em seguir as recomendações para o uso correto do AOC.

As características definidoras estudadas apresentaram sensibilidade, especificidade, valores preditivos positivos e negativos altos para o diagnóstico de enfermagem analisado.

Diante da elevada chance de ocorrer DE "Conhecimento Deficiente", por meio do "seguimento inadequado de instruções", ressalta-se a necessidade de rever as intervenções de Enfermagem para alcance de melhores resultados.

Pesquisas de intervenção deverão ser desenvolvidas com a finalidade de testar e/ou comparar estratégias de educação em saúde, gerando recursos que corroborem com aumento do conhecimento sobre uso correto do AOC.

3. Ministério da Saúde. Saúde sexual e saúde reprodutiva. Brasília (Brasil): Ministério da Saúde; 2010. 300p. 4. Dragoman MV. The combined oral contraceptive pill - recent developments, risks and benefits. Best Pract Res Clin Obstet Gynaecol.[Internet].2014 aug [acesso em: 18 jun 2015];28(6):825-834. Disponível em:

http://www.ncbi.nlm.nih.gov/pubmed/25028259

5. Petersen R, Albright J, Garrett JM, Curtis KM. Pregnancy and STD Prevention Couseling Using na Adaptation of Motivacional 
Interviewing: a randomized controlled trial. Perspect Sex Reprod Health. [Internet]. 2007 mar [acesso em: 19 jun 2015];39(1):21-28. Disponível em:

http://www.ncbi.nlm.nih.gov/pubmed/17355378 6. Ayoola AB, Zandee GL, Johnson E, Pennings K.Contraceptive use among low-income women living in medically underserved neighborhoods. J Obstet Gynecol Neonatal Nurs. [Internet]. 2014 jul-aug [acesso em: 18 jun 2015];43(4):455-464. Disponível em:

http://www.ncbi.nlm.nih.gov/pubmed/24958447

7. Organização Mundial de Saúde. Critérios Médicos de Elegibilidade para o uso de Métodos Anticoncepcionais. 4a. ed. Genebra (Suíça): Organização Mundial de Saúde;2009. 133p. 8. Lira ALBC, Lopes MVO. Pacientes transplantados renais: análise de associação dos diagnósticos de enfermagem. Rev. Gaúcha Enferm. [Internet]. 2010 [acesso em: $10 \mathrm{fev}$ 2014];31(1):108-114. Disponível em: http://dx.doi.org/10.1590/S1983-14472010000100015. 9. North American Nursing Diagnosis Association - (NANDA). Diagnósticos de enfermagem da NANDA: definições e classificação 2012-2014. Trad. de Jeanne L. M. Michel. Porto Alegre (Brasil): Artmed; 2012.

10. Carpenito-Moyet LJ. Manual de diagnósticos de enfermagem. 11a ed. Porto Alegre (RS): Artmed; 2008. 11. Lopes MVO, Silva VM, Araujo TL. Methods for Establishing the Accuracy of Clinical Indicators in Predicting Nursing Diagnoses.InternationalJournalofNursingKnowledge. 2012 oct; 23(3):134-9.

12. Ministério da Educação. Educação tem o maior avanço nos últimos 20 anos [Internet]. Brasília: Ministério da Educação; 2013 [acesso em: 12 nov 2013]. Disponível em:

http://portal.mec.gov.br/index.php?option=com_content\&vie $\underline{w}=$ article\&id=18927:educacao-tem-o-maior-avanco-nosultimos-20-anos\&catid $=222 \&$ ltemid $=86$

13. Américo CF, Nogueira PSF, Vieira RPRV, Bezerra CG, Moura ERFM, Lopes MVO. Knowledge of users of low-dose oral combined contraceptives about the method. Rev. Latino-Am. Enfermagem [Internet]. 2013 [acesso em: 13 jan 2013];21(4):928-34.Disponível em: http://dx.doi.org/10.1590/S010411692013000400014 14. Nicolau AIO, Dantas RC, Gadelha APP, Pinheiro AKB. Conhecimento, atitude e prática de mulheres residentes no meio rural acerca dos métodos contraceptivos. Rev. Eletr. Enf. [Internet]. 2012 jan-mar [acesso em 19 jun 2015];14(1):164170. Disponível em:

http://www.fen.ufg.br/revista/v14/n1/v14n1a19.htm 15. Hall KS, Castaño PM, Stone PW, Westhoff C. Measuring oral contraceptive knowledge: a review of research findings and limitations. PatientEducandCouns. [Internet]. 2010 [acesso em: 10 fev 2014];81(3): 388-394. Disponível em:

http://www.ncbi.nlm.nih.gov/pmc/articles/PMC3785081/

16. Secretaria de Assuntos Estratégicos da Presidência da República (SAE) [Internet]. Brasília: Secretaria de Assuntos Estratégicos (BR) [acesso em: $10 \mathrm{dez} 2013$ ]. Veja diferenças entre conceitos que definem classes sociais no Brasil. Disponível em:

http://www.sae.gov.br/site/?p=17821\#ixzz35z5EjCGj
17. Moura ERF, Silva RMS, Gomes AMA, Almeida PC, Evangelista DR. Perfil demográfico, socioeconômico e de saúde reprodutiva de mulheres atendidas em planejamento familiar no interior do Ceará. Revista Baiana de Saúde Pública. 2010; 34(1): 119-133.

18. Bahamondes L, Pinho F, Melo NR, Oliveira E, Bahamondes MV. Fatores associados à descontinuação do uso de anticoncepcionais orais combinados. Rev. Bras. Ginecol. Obstet. 2011;33(6):303-09.

19. White K, Hopkins K, Potter JE, Grossman D. Knowledge and attitudes about long-acting reversible contraception among Latina women who desire sterilization. Womens Health Issues. [Internet]. 2013 jul-aug [acesso em: 2015 jun 18];23(4):e257e263 Disponível em:

http://www.ncbi.nlm.nih.gov/pmc/articles/PMC3707629/ 20. Ramathuba DU, Khoza LB, Netshikweta ML. Knowledge, attitudes and practice of secondary school girls towards contraception in Limpopo Province. Curationis [Internet]. 2012 [acesso em: $31 \mathrm{dez}$ 2015];35(1):45. Disponível em: http://dx.doi.org/10.4102/curationis.v35i1.45.

21. Sousa MCR, Gomes KRO. Objective and perceived knowledge of oral contraceptive methods among adolescent mothers.Cad Saúde Pública. [Internet]. 2009 mar [acesso em: 10 jan 2014];25(3):645-54. Disponível em: http://dx.doi.org/10.1590/S0102-311X2009000300019.

22. Iftikhar R, Abdulrahman B, Khail A A. Knowledge about missed contraceptive pills among married women at King Abdulaziz University hospital. PatientPreferAdherence. [Internet]. 2015 [acesso em: 20 jun 2015];9:401-411. Disponível em: http://www.ncbi.nlm.nih.gov/pmc/articles/PMC4362980/ 23. Vogt $C$, Schaefer M. Knowledge matters--impact of two types of information brochure on contraceptive knowledge, attitudes and intentions. Eur J Contracept Reprod Health Care.[Internet]. 2012 apr [acesso em 2015 jun 18];17(2):13543. Disponível em:

http://www.ncbi.nlm.nih.gov/pubmed/22200343

24. Nunes WBN, Andrade LDF, Trigueiro JVS, Santos NCCB. Investigação das ações de enfermagem no planejamento familiar em cidades do curimataú paraibano. Rev. Ciênc. Saúde Nova Esperança. [Internet]. 2013 jun [acesso em 2015 jun 18];11(1):31-45. Disponível em:

http://www.facene.com.br/wpcontent/uploads/2010/11/Revista-Facene-Julho-2013.pdf 25. Zarate TM, Diaz-Martín T, Martínez-Astorquiza-Corral T.Evaluation of factors associated with noncompliance in users of combined hormonal contraceptive methods: a crosssectional study: results from the MIA study.BMC Womens Health.[Internet]. 2013 out [acesso em: 19 jun 2015]; 13:13-38. Disponível em:

http://www.ncbi.nlm.nih.gov/pmc/articles/PMC4015733/

Recebido: 04/09/2014.

Aceito: $10 / 06 / 2015$.

Publicado: 31/12/2015. 